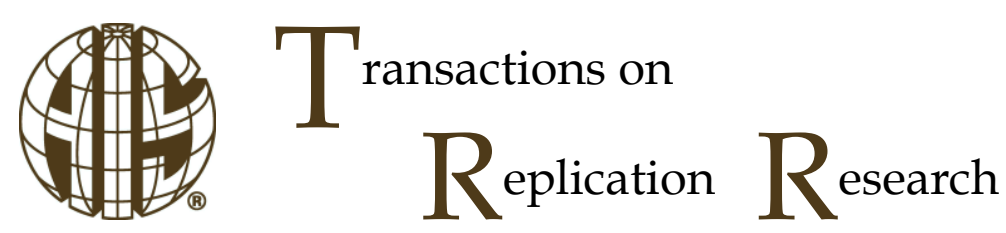

Conceptual Replication

ISSN 2473-3458

\title{
One Question, Two Answers: Mixed Findings of Information Technology Capability and Firm Performance and Their Implications
}

\author{
Inmyung Choi \\ Department of Supply Chain and Information Systems \\ lowa State University \\ ichoi@iastate.edu
}

\author{
Joey F. George \\ Department of Supply Chain and Information Systems \\ lowa State University \\ jfgeorge@iastate.edu
}

\begin{abstract}
:
This study conducted a conceptual replication on Chae et al. (2014) by utilizing multiple comparison groups of IT leading firms. Empirical testing for the positive association between information technology (IT) capability and firm performance has been a celebrated debate in IS research due to mixed findings; Bharadwaj (2000) and Santhanam and Hartono (2003) confirmed the positive impact of IT capability on firm performance, but Chae et al. (2014) suggested no relationship between the IT capability and performance. Understanding what produces contradictory results is a timely and critical issue because a large body of the business value of IS research has employed IT capability as a key construct. Whereas Chae et al. (2014) investigated the link between IT capability and firm performance by comparing the performance of an IT leader and that of a single matched group from 2001-2004, this study examined the relationship by building multiple comparison groups, which include all firms in the same industry. As a result, contrary to findings by Chae et al. (2014), this research indicated that IT capability has a significant impact on a firm's financial performance.
\end{abstract}

Keywords: IT capability, Firm performance, IT strategy, Business value of IT, InformationWeek 500

The manuscript was received 03/22/2016 and was with the authors 1 months for 1 revision.

\section{Introduction}

"The questions stay the same, only the answers change." (Stiglitz, 1989, p. 23)

Stiglitz's joke encapsulates what has happened to economics theories but is not only applicable to economic phenomena. The information systems (IS) research area also has a controversial and unsolved question: Does IT capability improve firm performance? Three MIS Quarterly papers have provided two different answers in the past two decades. First, the IT capability construct was defined and measured based on the data retrieved from the InformationWeek (IW) 500 list from 1991-1994 (Bharadwaj, 2000). A matched sample comparison was conducted by selecting leading IT groups from the IW 500 list as well as benchmark (or control) groups, whose revenue was similar (within $70 \%-130 \%$ of the IT leaders' revenue) to the IT leading groups but that were not on the IW 500 list. The IT leaders realized superior firm performance as measured by several accounting ratios. Several years later, the same issue was investigated, but a different comparison method was employed (Santhanam and Hartono, 2003). Whereas Bharadwaj (2000) selected a single benchmark group of leading IT firms, Santhanam and Hartono (2003) considered multiple benchmark groups of leading IT firms in the same industry to minimize selection bias. In other words, they compared the performance of IT leaders, such as Wal-Mart Stores, Inc., and the average performance of leading non-IT firms in the retail industry. The results of the second study confirmed the findings of the original investigation and revealed the robustness of the relationship between IT capability and firm performance. IT capability enhances firms' financial performance, and this influence is also sustained three years later. Until recently, the contribution of IT capability on firm 
performance has been accepted as true, and the IT capability construct has been the main pillar of the business value of IS papers (Stoel and Muhanna, 2009; Tanriverdi and Uysal, 2015; Wang et al., 2012).

Recently, Chae et al. (2014) suggested that IT capability does not have any positive influence on firm performance, and there is also no sustained impact of IT capability on the performance. The central tenet of this argument is that IT resources are widely available in markets, and they are commoditized after the prevalent use of enterprise resource planning (ERP) systems and Web technologies (Wang, 2010). Thus, it is difficult to develop superior IT capability from the deployment of IT systems without improving the managerial ability to use IT and having better IT systems does not necessarily enhance firm performance. To support their argument, Chae et al. (2014) replicated the study by Bharadwaj (2000). They analyzed more recent data (2001-2004) from the IW 500 compared to Bharadwaj (2000) and Santhanam and Hartono (2003), whose investigations used data from the IW 500 conducted in the early 1990s (19911994). Chae et al. (2014) developed a single matched group of IT leaders following Bharadwaj (2000). Notably, there was no significant difference between the financial performance of IT leaders and their competing firms, while the role of IT capability in the competitive landscape of firms had largely vanished. However, this may be a hasty generalization considering that Chae et al. (2014) did not compare the performance of IT leaders with that of all firms in the same industry, and some sample firms are not suitable to be considered benchmark firms. Figure 1 illustrates the differences in the three prior studies on IT capability and firm performance and this research.

\begin{tabular}{|c|c|c|c|}
\hline \multirow{3}{*}{ 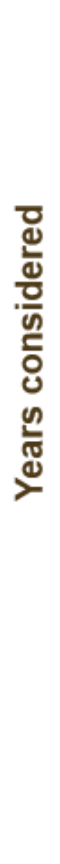 } & $\begin{array}{l}1991 \\
-1994\end{array}$ & $\begin{array}{c}\text { Financial performance of } \\
\text { IT leading group } \\
> \\
\text { Financial performance of } \\
\text { Control group }\end{array}$ & $\begin{array}{c}\text { Santhanam and } \\
\text { Hartono (2003) } \\
\text { Financial performance of } \\
\text { IT leading group } \\
> \\
\text { Financial performance of } \\
\text { Control group }\end{array}$ \\
\hline & \multirow[t]{2}{*}{$\begin{array}{l}2001 \\
-2004\end{array}$} & $\begin{array}{l}\text { Chae et al. (2014) } \\
\text { Financial performance of } \\
\text { IT leading group } \\
\approx \\
\text { Financial performance of } \\
\text { Control group }\end{array}$ & \begin{tabular}{|} 
This study \\
Financial performance of \\
IT leading group \\
$>$ \\
Financial performance of \\
Control group
\end{tabular} \\
\hline & & $\begin{array}{l}\text { A matched firm } \\
\text { Control }\end{array}$ & $\begin{array}{l}\text { All firms in the same } \\
\text { industry } \\
\text { groups }\end{array}$ \\
\hline
\end{tabular}

Figure 1. Comparison of This Study with Three Prior Studies

The purpose of this study is to fill in the gap which is shown in Figure 1 by replicating the results of Chae et al. (2014) using the IW 500 list from 2001-2004 along with multiple benchmark groups, as suggested by Santhanam and Hartono (2003). This investigation differs from the prior three studies in that we built the multiple comparison groups by selecting all firms in the same industry (two-digit and four-digit SIC codes were adopted) to which leading IT firms belong, and we used data from the early 2000s. In answering the research question of the current study, "Does IT capability have a positive impact on current and sustained firm performance?" this work will contribute to the IS research area in several ways. First, we suggest that the positive association between IT capability and firm performance still exists. Second, we propose that the use of multiple comparison groups, considering all firms in the same industry, is preferable in empirical research in IS. Third, we provide a paradigmatic case for why replication studies in IS are required and necessary for expanding our understanding of a certain topic. 
The remainder of this study is organized as follows. In the next section, the hypotheses are introduced. The research methods and results are presented, and then the implications of the study are discussed.

\section{Hypotheses}

We tested the eight hypotheses that Bharadwaj (2000), Santhanam and Hartono (2003), and Chae et al. (2014) examined.

Hypothesis 1: The average profit ratios of firms that have superior IT capability are higher than the average profit ratios of all other firms in the same industry.

Hypothesis 2: The average cost ratios of firms that have superior IT capability are lower than the average cost ratios of all other firms in the same industry.

Hypothesis 3: The average profit ratios of firms that have superior IT capability are higher than the average profit ratios of all other firms in the same industry over three subsequent years.

Hypothesis 4: The average cost ratios of firms that have superior IT capability are lower than the average cost ratios of all other firms in the same industry over three subsequent years.

Hypothesis 5: After controlling for prior financial performance, the average profit ratios of firms that have superior IT capability are higher than the average profit ratios of all other firms in the same industry.

Hypothesis 6: After controlling for prior financial performance, the average cost ratios of firms that have superior IT capability are lower than the average cost ratios of all other firms in the same industry.

Hypothesis 7: After controlling for prior financial performance, the average profit ratios of firms that have superior IT capability are higher than the average profit ratios of all other firms in the same industry over three subsequent years.

Hypothesis 8: After controlling for prior financial performance, the average cost ratios of firms that have superior IT capability are lower than the average cost ratios of all other firms in the same industry over three subsequent years.

\section{Research Method}

\subsection{Sample Selection}

This study precisely followed the procedure carried out in the prior three relevant studies (Bharadwaj, 2000; Chae et al., 2014; Santhanam and Hartono, 2003) to identify IT leaders in the IW 500 lists from 2001-2004. IW 500 list is the cornerstone of this research and three prior studies to assess a firm's IT capability, and we have examined several issues of using IW 500 list in Appendix A. During the period, the magazine announced 500 firms with a higher level of IT capability based on the assessment of editors in InformationWeek. The first step involved collecting data by building potential IT leaders appearing on the IW 500 list. In total, 2,000 firms were identified. In the second step, we chose firms that were listed more than once to develop a robust sample of IT leaders (Bharadwaj, 2000). For instance, Pfizer was not included in the leading IT group because the firm was listed only once on the IW 500 list in 2001. On the other hand, Wal-Mart Stores was regarded as an IT leader since the company was listed on the list from 2001-2003. After this step, 549 IT leaders in several industries were incorporated. In the third step, the comparison group of 549 IT leaders was assembled, and 337 industries were identified according to the standard industry classification (SIC) scheme. This study used the two-digit SIC code and the four-digit SIC code to build a comparison group. If an IT leader was the sole company in its industry, it was excluded in this step. After matching IT leaders with their rival firms, a total of 337 firms remained. These steps are summarized in Table 1.

\begin{tabular}{|c|c|c|}
\hline \multicolumn{3}{|c|}{ Table 1. Three Steps of Collecting Sample Data } \\
\hline Step & Procedure & Number of firms \\
\hline
\end{tabular}




\begin{tabular}{|c|l|c|}
\hline 1 & Collecting firms' data on the InformationWeek 500 lists from 2001 to 2004 & 2,000 \\
\hline 2 & Identifying the IT leaders, which appeared in the lists more than once & 549 \\
\hline 3 & Pairing the IT leaders with control groups & 337 \\
\hline
\end{tabular}

The biggest difference between this study and prior works (Bharadwaj, 2000; Chae et al., 2014) was that this research considered all firms in the same industry, whereas the prior two studies chose a single firm as the control group. When Bharadwaj (2000) and Chae et al. (2014) selected a control group of an IT leader, the average sales volume of the control firm was required to be within $70 \%-130 \%$ of that of an IT leader. In their research, four-digit SIC codes were initially adopted to build a control group, but two-digit codes were also used when there was no control group that satisfied the above requirement. In contrast, Santhanam and Hartono (2003) considered all firms in the same industry as the comparison group of an IT leader. This research follows the approach of Santhanam and Hartono (2003) because selecting a single control group can be arbitrary and reduces sample sizes.

\subsection{Research Method}

As prior investigations (Bharadwaj, 2000; Chae et al., 2014; Santhanam and Hartono, 2003) employed a paired matching comparison to test Hypotheses 1-4, this study followed the same procedure. Table 2 summarizes the eight profit ratios and cost ratios of the leading IT groups and comparison groups. Two statistical methods were adopted: pairwise t-test and Wilcoxon signed-rank test. The pairwise t-test assesses the difference between two groups (leading IT groups and their comparison group) by comparing the mean value of paired samples. Additionally, the Wilcoxon signed-rank test, a nonparametric statistical test used when comparing two matched samples, was conducted in this research because the Wilcoxon signed-rank test produces more robust results than pairwise t-test in samples that are not normally distributed (Bharadwaj, 2000).

\begin{tabular}{|c|c|l|}
\hline \multirow{2}{*}{ Table 2. Financial Ratios for Profit and Cost } \\
\hline Dependent variables & Ratios & \multicolumn{1}{c|}{ Definition } \\
\hline \multirow{4}{*}{ Profit ratios } & ROA & Net Income/ Total Assets \\
\cline { 2 - 3 } & ROS & Net Income/ Sales \\
\cline { 2 - 3 } & Ol/A & Operating Income/ Total Assets \\
\cline { 2 - 3 } & Ol/S & Operating Income/ Sales \\
\cline { 2 - 3 } & Ol/E & Operating Income/ Number of Employees \\
\hline \multirow{4}{*}{ Cost ratios } & COG/S & Cost of Goods Sold/ Sales \\
\cline { 2 - 3 } & SGA/S & Selling and General Administration Expenses/ Sales \\
\cline { 2 - 3 } & OPEXP/S & Operating Expense/ Sales \\
\hline
\end{tabular}

To evaluate the possible halo effect, concerned with Hypotheses 5-8, this study adopted two regression models:

1. $\quad$ Financial Performance ${ }_{t}=\alpha_{0}+\alpha_{1}$ Financial Performance $_{t-1}$

2. Financial Performance ${ }_{t}=\beta_{0}+\beta_{1}$ Financial Performance $_{t-1}+\beta_{2}$ ITC where $t=2002-2007$.

The financial performance variables are profit ratios and cost ratios, and the IT capability (ITC) variable is a dummy. ITC variables are coded as 1 for leading IT firms. On the other hand, ITC variables are coded as 0 for comparison groups. The regression model 1 tests whether the financial performance of a firm in a specific year was related to the prior year's financial performance. Statistically significant coefficient $\alpha_{1}$ indicates that the prior year's performance has a strong relationship with the current year's performance (Fama and French, 2000). The regression model 2 retests the association among financial measures but also evaluates the impact of IT capability on firm performance. If $\beta_{2}$ is statistically significant, then we can affirm that the IT capability has a sizable impact on firm performance. On the other hand, if $\beta_{1}$ is significant, but $\beta_{2}$ is not significant, it is difficult to deny the existence of a halo effect in selecting the leading IT firms. In other words, the firm in IW 500 was selected due to its prior financial performance rather than its IT capability. 


\section{Research Result}

\subsection{A Comparison of Financial Performance (H1 and H2)}

The results of the pairwise t-test (p-value) and Wilcoxon signed-rank test (z-value) for the 2001-2004 data are summarized in Table 3. In most cases, IT leaders realized better financial performances in profit ratios and lower values in cost ratios. In profit ratios (i.e., ROA, ROS, OI/A, OI/S, and OI/E), the mean and median values of IT leaders were higher than those of control groups. On the other hand, for cost ratios (i.e., $\mathrm{COG} / \mathrm{S}$, SGA/S, and OPEXP/S), the mean and median values of IT leaders were lower than those of the control groups. Exceptionally, there was no significant difference in Ol/E ratios from 2002-2004 when considering paired t-test results, a finding that may be due to the small companies in the control group taking advantage of managing their employees. Overall, this paper argues that Hypotheses 1 and 2 were supported.

\begin{tabular}{|c|c|c|c|c|c|c|c|c|c|c|c|}
\hline \multirow[b]{3}{*}{ Ratios } & \multirow[b]{3}{*}{ Groups } & \multicolumn{10}{|c|}{2001} \\
\hline & & \multicolumn{5}{|c|}{ Four-digit control group } & \multicolumn{5}{|c|}{ Two-digit control group } \\
\hline & & $\mathbf{N}$ & Mean & Median & $\begin{array}{c}\text { Wilcoxon } \\
\text { test }\end{array}$ & t-test & $\mathbf{N}$ & Mean & Median & $\begin{array}{l}\text { Wilcoxon } \\
\text { test }\end{array}$ & t-test \\
\hline \multirow{2}{*}{ ROA } & IT leaders & 337 & 0.0115 & 0.0261 & \multirow{2}{*}{$-13.690^{\star * *}$} & \multirow{2}{*}{$5.883^{* \star *}$} & 333 & .0115 & 0261 & \multirow{2}{*}{$-14.967^{\star \star *}$} & \multirow{2}{*}{$8.741^{\star \star \star}$} \\
\hline & Control & 337 & -0.6071 & -0.767 & & & 333 & -0.4171 & -0.3108 & & \\
\hline \multirow{2}{*}{ ROS } & IT leaders & 337 & 0.0179 & 0.0315 & \multirow{2}{*}{$-13.483^{* * *}$} & \multirow{2}{*}{$5.611^{* * *}$} & 333 & 0.0179 & 0.0315 & \multirow{2}{*}{$14.406^{\star * *}$} & \multirow{2}{*}{$6.265^{\star}$} \\
\hline & Control & 337 & -3.0458 & -0.1263 & & & 333 & -1.4229 & -0.3919 & & \\
\hline \multirow{2}{*}{$\mathrm{OI} / \mathrm{A}$} & IT leaders & 337 & 0.0742 & 0.0724 & \multirow{2}{*}{$-13.884^{\star * *}$} & \multirow{2}{*}{$5.421^{\star \star \star}$} & 333 & 0.0742 & 0.0724 & \multirow{2}{*}{$-14.828^{\star \star \star}$} & \multirow{2}{*}{$9.996^{\star \star \star}$} \\
\hline & Control & 337 & -0.4310 & -0.0203 & & & 333 & -0.2501 & -0.2219 & & \\
\hline \multirow{2}{*}{$\mathrm{Ol} / \mathrm{S}$} & IT leaders & 337 & 0.0995 & 0.0811 & \multirow{2}{*}{$-13.554^{* * *}$} & \multirow{2}{*}{$5.268^{\star * *}$} & 333 & 0.0995 & 0.0811 & \multirow{2}{*}{$-14.106^{* * *}$} & \multirow{2}{*}{$5.787^{\star \star \star}$} \\
\hline & Control & 337 & -2.5012 & -0.0348 & & & 333 & -1.3257 & -0.3795 & & \\
\hline \multirow{2}{*}{$\mathrm{Ol} / \mathrm{E}$} & IT leaders & 326 & 44.8777 & 18.7354 & \multirow{2}{*}{$-8.615^{\star * *}$} & \multirow{2}{*}{$3.745^{\star \star \star}$} & 322 & 44.8777 & 18.7354 & & \\
\hline & Control & 326 & 13.4222 & 3.8424 & & & 322 & 42.1916 & -4.6522 & & \\
\hline$c 0$ & IT leaders & 337 & 0.6830 & 0.7242 & & & 333 & 0.6830 & 0.7242 & $1 * * *$ & \\
\hline & Control & 337 & 2.2478 & 0.7985 & 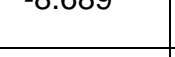 & & 333 & 1.3818 & 0.7747 & 10.001 & \\
\hline 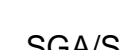 & IT leaders & 337 & 0.1638 & 0.1459 & & & 333 & 0.1638 & 0.1459 & & \\
\hline STH/S & Control & 337 & 1.0511 & 0.2558 & 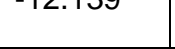 & & 333 & 0.8246 & 0.2978 & -1 & \\
\hline OPEXP & IT leaders & 337 & 0.8468 & 0.8768 & & & 333 & 0.8468 & 0.8768 & & \\
\hline & Control & 337 & 3.2989 & 0.9982 & & & 333 & 2.2064 & 1.2515 & & \\
\hline & & & & & & 0 & 02 & & & & \\
\hline & & & Fo & ticon & roup & & & & it c & Jroup & \\
\hline Ratios & Groups & $\mathbf{N}$ & Mean & Median & $\begin{array}{c}\text { Wilcoxon } \\
\text { test }\end{array}$ & t-test & $\mathbf{N}$ & Mean & Median & $\begin{array}{c}\text { Wilcoxon } \\
\text { test }\end{array}$ & t-test \\
\hline ROA & IT leaders & 337 & 0.0228 & 0.0273 & & & 333 & 0.0228 & 0.0273 & ** & \\
\hline & Control & 337 & -1.7892 & -0.0410 & & & 333 & -8.1433 & -0.2376 & & \\
\hline ROS & IT leaders & 337 & 0.0236 & 0.0355 & & & 333 & 0.0236 & 0.0355 & & \\
\hline & Control & 337 & -2.3910 & -0.0681 & & 0.0 & 333 & -1.5463 & -0.4741 & -14 & 0.000 \\
\hline 0 & IT leaders & 337 & 0.0771 & 0.0724 & & & 333 & 0.0771 & 0.0724 & & \\
\hline 政 & Control & 337 & -1.6636 & 0.0115 & 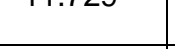 & & 333 & -5.9585 & -0.1782 & (דטסט & וט \\
\hline & IT leaders & 337 & 0.1051 & 0.0838 & & & 333 & 0.1051 & 0.0838 & & \\
\hline & Control & 337 & -1.7338 & 0.0086 & & & 333 & -1.0558 & -0.1778 & & \\
\hline $\mathrm{Ol} / \mathrm{E}$ & IT leaders & 334 & 74.5544 & 20.1606 & $-8.684^{\star * *}$ & -0.247 & 330 & 74.5544 & 20.1606 & $-5.275^{\star \star \star}$ & 0.437 \\
\hline
\end{tabular}




\begin{tabular}{|c|c|c|c|c|c|c|c|c|c|c|c|}
\hline \multicolumn{12}{|c|}{ Table 3. Results of Tests for Hypotheses 1 and 2} \\
\hline & Control & 334 & 86.2548 & 6.2673 & & & 330 & 60.9632 & 5.4945 & & \\
\hline \multirow{2}{*}{ COGS/S } & IT leaders & 337 & 0.6728 & 3 & \multirow{2}{*}{$1.589^{\star \star \star}$} & \multirow{2}{*}{$-2.613^{* * *}$} & 333 & 6728 & 0.7153 & \multirow{2}{*}{$-8.389^{* * *}$} & \multirow{2}{*}{$-4.266^{\star \star \star}$} \\
\hline & Control & 337 & 1.9096 & 0.7658 & & & 333 & 1.3659 & 0.7712 & & \\
\hline \multirow{2}{*}{ SGA/S } & IT leaders & 337 & 0.1694 & 0.1495 & \multirow{2}{*}{$-10.215^{\star * *}$} & \multirow{2}{*}{$-4.518^{* * *}$} & 333 & 0.1694 & 0.1495 & \multirow{2}{*}{$-12.746^{* \star *}$} & \multirow{2}{*}{$-5.913^{\star \star \star}$} \\
\hline & Control & 337 & 0.7220 & 0.2423 & & & 333 & 0.6746 & 0.3414 & & \\
\hline \multirow{2}{*}{$\begin{array}{c}\text { OPEXP } \\
\text { /S }\end{array}$} & IT leaders & 337 & 0.8422 & 0.8748 & \multirow{2}{*}{$.944^{* \star *}$} & \multirow{2}{*}{$-3.511^{* \star \star}$} & 333 & 0.8422 & 0.8748 & \multirow{2}{*}{$-14.041^{\star * *}$} & \multirow{2}{*}{$-5.374^{\star \star \star}$} \\
\hline & Control & 337 & 2.6318 & 0.9437 & & & 333 & 2.0407 & 1.1241 & & \\
\hline & & \multicolumn{10}{|c|}{2003} \\
\hline & & \multicolumn{5}{|c|}{ Four-digit control group } & \multicolumn{5}{|c|}{ Two-digit control group } \\
\hline Ratios & Groups & $\mathbf{N}$ & $\mathbf{N}$ & Median & \begin{tabular}{|c|}
$\begin{array}{c}\text { Wilcoxon } \\
\text { test }\end{array}$ \\
\end{tabular} & est & $\mathbf{N}$ & $n$ & Median & \begin{tabular}{|c|}
$\begin{array}{c}\text { Wilcoxon } \\
\text { test }\end{array}$ \\
\end{tabular} & t-test \\
\hline \multirow{2}{*}{$\mathrm{ROA}$} & IT leaders & 337 & 0.0349 & 0.0333 & \multirow{2}{*}{$-12.035^{\star * *}$} & \multirow{2}{*}{$2.378^{* *}$} & 337 & 0.0349 & 0.0333 & & \\
\hline & Control & 337 & -4.7785 & -0.0601 & & & 337 & -5.6342 & -0.2225 & & \\
\hline & IT leaders & 337 & 0.0481 & 0.0446 & & & 337 & 0.0481 & 0.0446 & & \\
\hline RUS & Control & 337 & -1.2405 & -0.0336 & & & 337 & -0.6600 & -0.3679 & & \\
\hline & IT leaders & 337 & 0.08 & 0 & & & 337 & 0.0810 & 738 & & \\
\hline 4 & Control & 337 & -1.5414 & 0.0051 & & & 337 & -2.3637 & -0.1774 & -15.010 & 1.10 \\
\hline Sו & IT leaders & 337 & 0 & 2 & & & 337 & 1141 & 0.0862 & & \\
\hline (1/3 & Control & 337 & -1.1232 & 0.0054 & & & 337 & -0.6111 & -0.1277 & & \\
\hline & IT leaders & 335 & 83.9498 & 22.7162 & & & 335 & 83.9498 & 22.7162 & & \\
\hline (1/L & Control & 335 & 63.0799 & 7.3867 & & & 335 & 81.3437 & 4.5113 & & \\
\hline & IT leaders & 337 & 0.6667 & 0.7200 & & & 337 & 0.6667 & 0.7200 & & \\
\hline . & Control & 337 & 1.4186 & 0.7652 & & & 337 & 1.0232 & 0.7823 & & \\
\hline & IT leaders & 337 & 0.1693 & 0.1507 & & & 337 & 0.1693 & 0.1507 & & \\
\hline$S G A / S$ & Control & 337 & 0.6270 & 0.2563 & & & 337 & 0.5191 & 0.3044 & & \\
\hline$X P$ & IT leaders & 337 & 0.8361 & 0.8749 & & & 337 & 0.8361 & 0.8749 & & \\
\hline & Control & 337 & 2.0456 & 0.9614 & & & 337 & 1.5422 & 1.0334 & & \\
\hline & & & & & & 20 & 04 & & & & \\
\hline & & & Fou & $\mathrm{r}-$ & oup & & & Two & co & oup & \\
\hline Ratios & Groups & $\mathbf{N}$ & Mean & Median & $\begin{array}{c}\text { Wilcoxon } \\
\text { test }\end{array}$ & t-test & $\mathbf{N}$ & Mean & Median & $\begin{array}{c}\text { Wilcoxon } \\
\text { test }\end{array}$ & t-test \\
\hline & aders & 337 & & & & & 337 & & & & \\
\hline$K \cup A$ & Control & 337 & -0.6389 & 0.0025 & -10.910 & & 337 & -0.9270 & -0.3047 & -10.119 & \\
\hline$R_{2}>$ & IT leaders & 337 & 0.0576 & 0.0532 & & & 337 & 0.0576 & 0.0532 & & \\
\hline nus & Control & 337 & -1.9733 & 0.0006 & & & 337 & -1.8314 & -0.1789 & & \\
\hline & IT leaders & 337 & 0.0906 & 0.0813 & & & 337 & 0.0906 & 0.0813 & & \\
\hline & Control & 337 & -0.4518 & 0.0161 & & & 337 & -0.7206 & -0.1252 & & \\
\hline & IT leaders & 337 & 0.1248 & 0.1029 & & & 337 & 0.1248 & 0.1029 & & \\
\hline $01 / 5$ & Control & 337 & -1.6184 & 0.0345 & -11.109 & & 337 & -1.7354 & -0.1317 & -13.858 & 2.05 \\
\hline & IT leaders & 334 & 83.4778 & 30.367 & & & 334 & 83.4778 & 30.3672 & & \\
\hline$U / / E$ & Control & 334 & 85.4285 & 12.989 & $-5 . / 81^{n}$ & -0.0 & 334 & 87.2151 & 10.9825 & -4 & \\
\hline & IT leaders & 337 & 0.6609 & 0.7161 & & & 337 & 0.6609 & 0.7161 & & \\
\hline & Control & 337 & 1.8141 & 0.7504 & & & 337 & 0.9690 & 0.7709 & & \\
\hline SGA/S & IT leaders & 337 & 0.1692 & 0.141 & $-10.875^{\star * *}$ & $-2.609^{\star * *}$ & 337 & 0.1692 & 0.1410 & $-13.119^{* * *}$ & $-2.003^{* *}$ \\
\hline
\end{tabular}




\begin{tabular}{|c|c|c|c|c|c|c|c|c|c|c|c|}
\hline \multicolumn{12}{|c|}{ Table 3. Results of Tests for Hypotheses 1 and 2} \\
\hline & Control & 337 & 1.2512 & 0.2356 & & & 337 & 1.6867 & 0.3185 & & \\
\hline \multirow{2}{*}{$\begin{array}{l}\text { OPEXP } \\
\text { /S }\end{array}$} & IT leaders & 337 & 0.8302 & 0.8655 & \multirow{2}{*}{$-11.926^{\star * *}$} & \multirow{2}{*}{$-3.922^{* *}$} & 337 & 0.8302 & 0.8655 & \multirow{2}{*}{$-14.127^{* * *}$} & \multirow{2}{*}{$-2.347^{\star \star}$} \\
\hline & Control & 337 & 3.0652 & 0.9536 & & & 337 & 2.6556 & 1.1490 & & \\
\hline
\end{tabular}

\subsection{A Comparison of Sustained Financial Performance ( $\mathrm{H} 3$ and $\mathrm{H} 4)$}

This study also examined the sustained competitive advantage of IT leaders by comparing the financial performance of two groups (IT leaders and their control groups) measured from 2005-2007. If IT capability has an impact on firms' sustained competitiveness, the financial performances of IT leaders should be superior to those of the control groups. Profit ratios and cost ratios were used to identify the difference between the two groups in sustained financial performance, and the results are summarized in Table 4. The mean and median value of the profit ratios (i.e., ROA, ROS, OI/A, and OI/S) of the IT leaders were significantly higher than those of the control groups from 2005-2007. For the cost ratios (i.e., COG/S, SGA/S, and OPEXP/S), IT leaders had lower mean and median values compared to the control group. However, there was no significant difference between the OI/E of the IT leaders and the control groups. This study identified a substantial difference in the sustained financial performance between IT leaders and control groups. Subsequently, this study argued that Hypotheses 3 and 4 were supported.

\begin{tabular}{|c|c|c|c|c|c|c|c|c|c|c|c|}
\hline \multirow[b]{3}{*}{ Ratios } & \multirow[b]{3}{*}{ Groups } & \multicolumn{10}{|c|}{2005} \\
\hline & & \multicolumn{5}{|c|}{ Four-digit control group } & \multicolumn{5}{|c|}{ Two-digit control group } \\
\hline & & $\mathbf{N}$ & Mean & Median & $\begin{array}{c}\text { Wilcoxon } \\
\text { test }\end{array}$ & t-test & $\mathbf{N}$ & Mean & Median & $\begin{array}{c}\text { Wilcoxon } \\
\text { test }\end{array}$ & t-test \\
\hline \multirow{2}{*}{ ROA } & IT leaders & 324 & 0.0422 & 0.0454 & \multirow{2}{*}{$-12.061^{* * *}$} & \multirow{2}{*}{$5.425^{\star \star \star}$} & 324 & 0.0422 & 0.0454 & \multirow{2}{*}{$-14.316^{* * *}$} & \multirow{2}{*}{$5.981^{\star \star \star}$} \\
\hline & Control & 324 & -0.6582 & -0.0148 & & & 324 & -0.6012 & -0.1662 & & \\
\hline \multirow{2}{*}{ ROS } & IT leaders & 324 & 0.0542 & 0.0581 & \multirow{2}{*}{$-10.906^{* * *}$} & \multirow{2}{*}{$3.947^{* * *}$} & 324 & 0.0542 & 0.0581 & \multirow{2}{*}{$-13.513^{* * *}$} & \multirow{2}{*}{$5.040^{\star \star \star}$} \\
\hline & Control & 324 & -3.9071 & -0.0076 & & & 324 & -1.9060 & -0.1230 & & \\
\hline \multirow{2}{*}{$\mathrm{Ol} / \mathrm{A}$} & IT leaders & 324 & 0.0959 & 0.0918 & \multirow{2}{*}{$-11.442^{* \star *}$} & \multirow{2}{*}{$5.940^{* * *}$} & 324 & 0.0959 & 0.0918 & \multirow{2}{*}{$-13.954^{* * *}$} & \multirow{2}{*}{$6.398^{\star \star \star}$} \\
\hline & Control & 324 & -0.3651 & 0.0264 & & & 324 & -0.3855 & -0.0736 & & \\
\hline \multirow{2}{*}{$\mathrm{Ol} / \mathrm{S}$} & IT leaders & 324 & 0.1285 & 0.1098 & \multirow{2}{*}{$-11.359^{* * *}$} & \multirow{2}{*}{$3.675^{\star * \star}$} & 324 & 0.1285 & 0.1098 & \multirow{2}{*}{$-13.209^{* * *}$} & \multirow{2}{*}{$5.021^{\star \star \star}$} \\
\hline & Control & 324 & -3.0249 & 0.0390 & & & 324 & -1.5988 & -0.1788 & & \\
\hline \multirow{2}{*}{$\mathrm{Ol} / \mathrm{E}$} & IT leaders & 323 & 89.5065 & 31.1237 & \multirow{2}{*}{$-7.590^{* \star *}$} & \multirow{2}{*}{-0.863} & 323 & 89.5065 & 31.1237 & \multirow{2}{*}{$-3.719^{* \star *}$} & \multirow{2}{*}{-0.566} \\
\hline & Control & 323 & 214.5044 & 13.3355 & & & 323 & 104.6736 & 7.5332 & & \\
\hline COGS/ & IT leaders & 324 & 0.6645 & 0.7161 & $-5909^{* * *}$ & - 2930 & 324 & 0.6645 & 0.7161 & $-8695^{* * *}$ & -4517 \\
\hline $\mathrm{S}$ & Control & 324 & 3.0428 & 0.7402 & $-5.909^{\circ}$ & -2.930 & 324 & 1.6562 & 0.7535 & -8.695 & $-4.51 / r$ \\
\hline SGA/S & IT leaders & 324 & 0.1656 & 0.1386 & $10125^{\star \star \star}$ & -4087 & 324 & 0.1656 & 0.1386 & $12110^{* * *}$ & $2034^{* * *}$ \\
\hline & Control & 324 & 0.9615 & 0.2295 & $90.1<0^{2}$ & | T. Tor & 324 & 0.8976 & 0.2981 & - 12.170 & 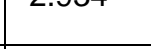 \\
\hline OPEXP & IT leaders & 324 & 0.8301 & 0.8612 & $-11386^{* * *}$ & & 324 & 0.8301 & 0.8612 & $-13,731^{* * *}$ & -5.04 \\
\hline 10 & Control & 324 & 4.0043 & 0.9334 & -11.386 & $\left.\right|^{-3}$ & 324 & 2.5538 & 1.1478 & $-13.73 \mid$ & -5.042 \\
\hline & & & & & & 20 & 06 & & & & \\
\hline & & & Four & -digit con & trol group & & & Two & -digit con & ntrol group & \\
\hline Ratios & Groups & $\mathbf{N}$ & Mean & Median & $\begin{array}{c}\text { Wilcoxon } \\
\text { test }\end{array}$ & t-test & $\mathbf{N}$ & Mean & Median & \begin{tabular}{|c}
$\begin{array}{c}\text { Wilcoxon } \\
\text { test }\end{array}$ \\
\end{tabular} & t-test \\
\hline BOA & IT leaders & 313 & 0.0543 & 0.0483 & $-10986^{* * *}$ & $2385^{\star *}$ & 313 & 0.0543 & 0.0483 & $-10735^{* * *}$ & $5180^{* * *}$ \\
\hline ה ח & Control & 313 & -0.8522 & 0.0068 & 90.000 & $2.000^{\circ}$ & 313 & -0.8822 & -0.0122 & 10.100 & 0.100 \\
\hline ROS & IT leaders & 313 & 0.0713 & 0.0597 & $-11067^{* * *}$ & 285 & 313 & 0.0713 & 0.0597 & -13 & \\
\hline 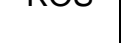 & Control & 313 & -3.2437 & 0.0068 & & 2.000 & 313 & -0.7253 & -0.1316 & & \\
\hline
\end{tabular}




\begin{tabular}{|c|c|c|c|c|c|c|c|c|c|c|c|}
\hline \multicolumn{12}{|c|}{ Table 4. Results of Tests for Hypotheses 3 and 4} \\
\hline \multirow{2}{*}{$\mathrm{Ol} / \mathrm{A}$} & IT leaders & 313 & 0.0971 & 0.0866 & \multirow{2}{*}{$-10.761^{\star * *}$} & \multirow{2}{*}{$3.106^{* * *}$} & 313 & 0.0971 & 0.0866 & \multirow{2}{*}{$-10.288^{\star * *}$} & \multirow{2}{*}{$5.151^{* * *}$} \\
\hline & Control & 313 & -0.4532 & 0.0434 & & & 313 & -0.7864 & 0.0274 & & \\
\hline \multirow{2}{*}{$\mathrm{Ol} / \mathrm{S}$} & IT leaders & 313 & 0.1308 & 0.1036 & \multirow{2}{*}{$-10.952^{\star \star *}$} & \multirow{2}{*}{$2.801^{* * *}$} & 313 & 0.1308 & 0.1036 & \multirow{2}{*}{$-12.304^{\star \star *}$} & \multirow{2}{*}{$9.801^{\star * \star}$} \\
\hline & Control & 313 & -2.6354 & 0.0496 & & & 313 & -0.5243 & -0.0427 & & \\
\hline \multirow{2}{*}{$\mathrm{Ol} / \mathrm{E}$} & IT leaders & 313 & 99.0485 & 35.3144 & \multirow{2}{*}{$-5.597^{\star \star \star}$} & \multirow{2}{*}{-1.067} & 313 & 99.0485 & 35.3144 & \multirow{2}{*}{$-2.898^{\star \star \star}$} & \multirow{2}{*}{-1.130} \\
\hline & Control & 313 & 145.3940 & 19.1163 & & & 313 & 128.3350 & 18.7935 & & \\
\hline \multirow{2}{*}{$\begin{array}{c}\text { COGS/ } \\
\mathrm{S}\end{array}$} & IT leaders & 313 & 0.6648 & 0.7184 & \multirow{2}{*}{$-5.426^{\star \star \star}$} & \multirow{2}{*}{$-2.395^{\star \star}$} & 313 & 0.6648 & 0.7184 & \multirow{2}{*}{$-7.756^{\star \star \star}$} & \multirow{2}{*}{$-7.057^{\star \star \star}$} \\
\hline & Control & 313 & 2.7891 & 0.7542 & & & 313 & 1.0756 & 0.7514 & & \\
\hline \multirow{2}{*}{ SGA/S } & IT leaders & 313 & 0.1667 & 0.1354 & \multirow{2}{*}{$-9.649^{\star * *}$} & \multirow{2}{*}{$-3.725^{\star \star \star}$} & 313 & 0.1667 & 0.1354 & \multirow{2}{*}{$-11.260^{* * *}$} & \multirow{2}{*}{$-7.627^{\star \star \star}$} \\
\hline & Control & 313 & 0.7223 & 0.2231 & & & 313 & 0.4077 & 0.2885 & & \\
\hline \multirow{4}{*}{$\begin{array}{c}\text { OPEXP } \\
\text { /S }\end{array}$} & IT leaders & 313 & 0.8315 & 0.8626 & $-11331^{* \star *}$ & & 313 & 0.8315 & 0.8626 & & \\
\hline & Control & 313 & 3.5113 & 0.9361 & -51.001 & & 313 & 1.4832 & 1.0281 & 1 & -5 \\
\hline & & & & & & 20 & 07 & & & & \\
\hline & & & Fol & digito & trol group & & & $\mathbf{T}$ & D-diait c & trol group & \\
\hline Ratios & Groups & $\mathbf{N}$ & $M$ & an & \begin{tabular}{|c|}
$\begin{array}{c}\text { Wilcoxon } \\
\text { test }\end{array}$ \\
\end{tabular} & est & $\mathbf{N}$ & Mean & Median & $\begin{array}{c}\text { Wilcoxon } \\
\text { test }\end{array}$ & t-test \\
\hline BOA & IT leaders & 296 & 0.0450 & 96 & & 5 & 296 & 0.0450 & 0.0496 & ** & \\
\hline ה ח & Control & 296 & -0.6679 & 0.0041 & 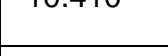 & 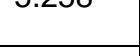 & 296 & -0.4603 & -0.1183 & -1 & 0.006 \\
\hline$B_{2}>$ & IT leaders & 296 & 0.0518 & 0.055 & & & 296 & 0.0518 & 0.0555 & & \\
\hline S & Control & 296 & -1.9124 & 0.0015 & 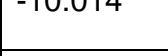 & & 296 & -0.9741 & -0.0622 & t & \\
\hline 0 & IT leaders & 296 & 0.0895 & 0.0882 & & & 296 & 0.0895 & 0.0882 & & \\
\hline U & Control & 296 & -0.4074 & 0.0403 & & & 296 & -0.2479 & 0.0127 & t & \\
\hline $\mathrm{O} / \mathrm{S}$ & IT leaders & 296 & 0.1192 & 0.1039 & *** & 4 & 296 & 0.1192 & 0.1039 & $\mid 4^{* \star *}$ & $1767-37$ \\
\hline 0 & Control & 296 & -1.3263 & 0.0461 & $0.0<1$ & | & 296 & -0.2479 & 0.0127 & 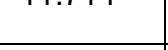 & 0.10 \\
\hline 0 & IT leaders & 296 & 92.7738 & 32.7278 & & & 293 & 92.7738 & 32.7278 & & \\
\hline I/L & Control & 296 & 185.1624 & 17.248 & 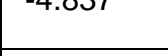 & .0 & 293 & 180.7033 & 15.1232 & -2.001 & -2.005 \\
\hline COGS/ & IT leaders & 296 & 0.6719 & 0.7057 & ** & & 296 & 0.6719 & 0.7057 & $55^{\star * * *}$ & $-7>-7$ \\
\hline & Control & 296 & 1.5545 & 0.7392 & T.0 & 00 & 296 & 1.0405 & 0.7555 & 1.100 & (1.01 \\
\hline$S$ & IT leaders & 296 & 0.1655 & 0.1381 & & & 296 & 0.1655 & 0.1381 & & \\
\hline S & Control & 296 & 0.5979 & 0.2177 & 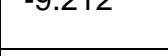 & -4.441 & 296 & 0.5465 & 0.2775 & 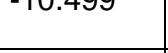 & -0 \\
\hline OPEXP & IT leaders & 296 & 0.8374 & 0.8616 & $x \times x \times$ & & 296 & 0.8374 & 0.8616 & $x \times x$ & \\
\hline & Control & 296 & 2.1524 & 0.9362 & $-10.0<0$ & $-4 .<00$ & 296 & 1.5870 & 0.9506 & 年 & 006 \\
\hline
\end{tabular}

\subsection{Testing for Halo Effect (H5-H8)}

Two regression models 1 and 2 were tested to assess whether the halo effect was present when selecting leading IT groups. The regression results are described in Table 5. In almost all cases, prior performance had a significant impact on firm performance. For instance, the coefficient of prior year financial performance on the ROA of 2002 in the four-digit column was 0.206 , which is significant at the $1 \%$ level. This means that the ROA of firms in 2002 was largely related to the ROA of firms in 2001. By the same token, the influence of IT capability on firm performance after controlling for prior firm performance can be estimated by the coefficient of IT capability. About two-thirds of the coefficients of IT capability in Table 5 were statistically significant at the $5 \%$ level. These results partially support the Hypotheses 5 and 6 in this study.

Table 5. Results of Tests for Hypotheses 5 and 6 


\begin{tabular}{|c|c|c|c|c|c|c|c|c|c|}
\hline \multirow[b]{3}{*}{ Ratios } & \multirow[b]{3}{*}{ Model } & \multicolumn{8}{|c|}{2002} \\
\hline & & \multicolumn{4}{|c|}{ Four-digit control group } & \multicolumn{4}{|c|}{ Two-digit control group } \\
\hline & & $\mathbf{N}$ & $R^{2}$ & $\begin{array}{c}\text { Prior year } \\
\text { financial } \\
\text { performance }\end{array}$ & $\begin{array}{c}\text { IT capability } \\
\text { dummy }\end{array}$ & $\mathbf{N}$ & $R^{2}$ & $\begin{array}{c}\text { Prior year } \\
\text { financial } \\
\text { performance }\end{array}$ & $\begin{array}{l}\text { IT capability } \\
\text { dummy }\end{array}$ \\
\hline \multirow{2}{*}{ ROA } & 1 & 674 & 0.043 & $0.206^{* * *}$ & & 670 & 0.000 & 0.010 & \\
\hline & 2 & 674 & 0.044 & $0.198^{* * *}$ & 0.037 & 670 & 0.046 & -0.062 & $0.226^{* * *}$ \\
\hline \multirow{2}{*}{ ROS } & 1 & 674 & 0.034 & $0.184^{* * *}$ & & 670 & 0.057 & $0.239^{\star \star \star}$ & \\
\hline & 2 & 674 & 0.044 & $0.162^{* * *}$ & $0.104^{* * *}$ & 670 & 0.084 & $0.199^{* * *}$ & $0.168^{* * *}$ \\
\hline \multirow{2}{*}{$\mathrm{OI} / \mathrm{A}$} & 1 & 674 & 0.031 & $0.175^{* * *}$ & & 670 & 0.000 & -0.011 & \\
\hline & 2 & 674 & 0.032 & $0.166^{* * \star *}$ & 0.042 & 670 & 0.033 & $-0.081^{* *}$ & $0.195^{\star \star *}$ \\
\hline \multirow{2}{*}{$\mathrm{Ol} / \mathrm{S}$} & 1 & 674 & 0.028 & $0.168^{* * *}$ & & 670 & 0.052 & $0.227^{\star \star *}$ & \\
\hline & 2 & 674 & 0.040 & $0.146^{\star * *}$ & $0.109^{\star * *}$ & 670 & 0.074 & $0.194^{\star * *}$ & $0.154^{* * *}$ \\
\hline \multirow{2}{*}{$\mathrm{Ol} / \mathrm{E}$} & 1 & 652 & 0.052 & $0.229^{\star * *}$ & & 667 & 0.883 & $0.939^{\star * *}$ & \\
\hline & 2 & 652 & 0.055 & $0.233^{\star * *}$ & -0.053 & 667 & 0.885 & $0.940^{\star * *}$ & $-0.044^{* * *}$ \\
\hline \multirow{2}{*}{$\begin{array}{c}\text { COGS/ } \\
\mathrm{S}\end{array}$} & 1 & 674 & 0.021 & $0.146^{\star \star *}$ & & 670 & 0.000 & 0.016 & \\
\hline & 2 & 674 & 0.028 & $0.134^{\star * *}$ & $-0.082^{* *}$ & 670 & 0.027 & -0.003 & $-0.164^{* * *}$ \\
\hline \multirow{2}{*}{ SGA/S } & 1 & 674 & 0.026 & $0.162^{\star \star *}$ & & 670 & 0.328 & $0.573^{\star \star *}$ & \\
\hline & 2 & 674 & 0.046 & $0.132^{\star \star \star}$ & $-0.144^{* \star *}$ & 670 & 0.333 & $0.553^{\star \star \star}$ & $-0.073^{* *}$ \\
\hline \multirow{4}{*}{$\begin{array}{l}\text { OPEXP } \\
\text { /S }\end{array}$} & 1 & 674 & 0.027 & $0.165^{\star * *}$ & & 670 & 0.042 & $0.204^{* * *}$ & \\
\hline & 2 & 674 & 0.038 & $0.145^{\star \star *}$ & $-0.106^{* * *}$ & 670 & 0.069 & $0.168^{* * *}$ & $-0.169^{* * *}$ \\
\hline & & \multicolumn{8}{|c|}{2003} \\
\hline & & \multicolumn{4}{|c|}{ Four-digit control group } & \multicolumn{4}{|c|}{ Two-digit control group } \\
\hline Ratios & Model & $\mathbf{N}$ & $R^{2}$ & $\begin{array}{c}\text { Prior year } \\
\text { financial } \\
\text { performance }\end{array}$ & $\begin{array}{c}\text { IT capability } \\
\text { dummy }\end{array}$ & $\mathbf{N}$ & $R^{2}$ & $\begin{array}{c}\text { Prior year } \\
\text { financial } \\
\text { performance }\end{array}$ & $\begin{array}{l}\text { IT capability } \\
\text { dummy }\end{array}$ \\
\hline \multirow{2}{*}{ ROA } & 1 & 674 & 0.000 & 0.008 & & 670 & 0.042 & $0.205^{* * *}$ & \\
\hline & 2 & 674 & 0.008 & 0.001 & $0.091^{* *}$ & 670 & 0.077 & $0.166^{* * *}$ & $0.191^{* * *}$ \\
\hline \multirow{2}{*}{ ROS } & 1 & 674 & 0.028 & $0.167^{* * *}$ & & 670 & 0.001 & 0.028 & \\
\hline & 2 & 674 & 0.068 & $0.138^{* * *}$ & $0.204^{* * *}$ & 670 & 0.081 & -0.035 & $0.290^{* * *}$ \\
\hline \multirow{2}{*}{$\mathrm{OI} / \mathrm{A}$} & 1 & 674 & 0.002 & 0.040 & & 670 & 0.042 & $0.204^{* * *}$ & \\
\hline & 2 & 674 & 0.016 & 0.031 & $0.121^{* * *}$ & 670 & 0.109 & $0.160^{* * *}$ & $0.264^{\star \star *}$ \\
\hline \multirow{2}{*}{$\mathrm{OI} / \mathrm{S}$} & 1 & 674 & 0.030 & $0.174^{\star * *}$ & & 670 & 0.000 & 0.012 & \\
\hline & 2 & 674 & 0.073 & $0.145^{\star \star *}$ & $0.208^{* * *}$ & 670 & 0.140 & $-0.063^{*}$ & $0.381^{* * *}$ \\
\hline \multirow{2}{*}{$\mathrm{Ol} / \mathrm{E}$} & 1 & 668 & 0.470 & $0.686^{\star \star \star}$ & & 667 & 0.965 & $0.982^{* \star *}$ & \\
\hline & 2 & 668 & 0.471 & $0.686^{\star \star *}$ & 0.030 & 667 & 0.965 & $0.983^{* * *}$ & $-0.013^{*}$ \\
\hline \multirow{2}{*}{$\begin{array}{c}\text { COGS/ } \\
\mathrm{S}\end{array}$} & 1 & 674 & 0.013 & $0.115^{\star \star \star}$ & & 670 & 0.001 & 0.024 & \\
\hline & 2 & 674 & 0.047 & $0.097^{\star *}$ & $-0.185^{\star \star \star}$ & 670 & 0.070 & -0.020 & $-0.268^{* \star *}$ \\
\hline \multirow{2}{*}{ SGA/S } & 1 & 674 & 0.022 & $0.148^{\star \star \star}$ & & 670 & 0.019 & $0.139^{\star \star *}$ & \\
\hline & 2 & 674 & 0.037 & $0.127^{\star \star \star}$ & $-0.124^{\star \star *}$ & 670 & 0.171 & 0.049 & $-0.400^{\star * *}$ \\
\hline \multirow{4}{*}{$\begin{array}{l}\text { OPEXP } \\
\text { /S }\end{array}$} & 1 & 674 & 0.024 & $0.156^{* \star *}$ & & 670 & 0.005 & $0.074^{*}$ & \\
\hline & 2 & 674 & 0.068 & $0.127^{\star \star *}$ & $-0.212^{\star \star \star}$ & 670 & 0.136 & -0.002 & $-0.370^{\star * *}$ \\
\hline & & & & & 200 & & & & \\
\hline & & & Four & igit control gro & & & & -digit control & roup \\
\hline
\end{tabular}




\begin{tabular}{|c|c|c|c|c|c|c|c|c|c|}
\hline \multicolumn{10}{|c|}{ Table 5. Results of Tests for Hypotheses 5 and 6} \\
\hline Ratios & Model & $\mathbf{N}$ & $R^{2}$ & $\begin{array}{c}\text { Prior year } \\
\text { financial } \\
\text { performance }\end{array}$ & $\begin{array}{c}\text { IT capability } \\
\text { dummy }\end{array}$ & $\mathbf{N}$ & $R^{2}$ & $\begin{array}{c}\begin{array}{c}\text { Prior year } \\
\text { financial } \\
\text { performance }\end{array} \\
\end{array}$ & $\begin{array}{l}\text { IT capability } \\
\text { dummy }\end{array}$ \\
\hline \multirow{2}{*}{$\mathrm{ROA}$} & 1 & 674 & 0.000 & 0.007 & & 674 & 0.000 & 0.016 & \\
\hline & 2 & 674 & 0.047 & -0.012 & $0.217^{* * *}$ & 674 & 0.064 & -0.042 & $0.259^{* * *}$ \\
\hline \multirow{2}{*}{ ROS } & 1 & 674 & 0.152 & $0.390^{* * *}$ & & 674 & 0.029 & $0.170^{* * *}$ & \\
\hline & 2 & 674 & 0.154 & $0.382^{* * *}$ & 0.038 & 674 & 0.032 & $0.155^{\star * \star}$ & 0.054 \\
\hline \multirow{2}{*}{$\mathrm{OI} / \mathrm{A}$} & 1 & 674 & 0.005 & $0.069^{*}$ & & 674 & 0.006 & $0.080^{\star *}$ & \\
\hline & 2 & 674 & 0.046 & 0.044 & $0.204^{* \star *}$ & 674 & 0.060 & 0.010 & $0.243^{* \star *}$ \\
\hline \multirow{2}{*}{$\mathrm{OI} / \mathrm{S}$} & 1 & 674 & 0.103 & $0.321^{* * *}$ & & 674 & 0.065 & $0.255^{\star * \star}$ & \\
\hline & 2 & 674 & 0.103 & $0.316^{* * *}$ & 0.023 & 674 & 0.065 & $0.255^{\star * *}$ & -0.001 \\
\hline \multirow{2}{*}{$\mathrm{Ol} / \mathrm{E}$} & 1 & 668 & 0.807 & $0.898^{\star \star *}$ & & 671 & 0.912 & $0.955^{\star \star \star}$ & \\
\hline & 2 & 668 & 0.807 & $0.899^{* * \star}$ & -0.025 & 671 & 0.912 & $0.955^{\star \star \star}$ & -0.008 \\
\hline \multirow{2}{*}{$\begin{array}{c}\mathrm{COGS} / \\
\mathrm{S}\end{array}$} & 1 & 674 & 0.399 & $0.631^{* \star *}$ & & 674 & 0.456 & $0.676^{\star \star *}$ & \\
\hline & 2 & 674 & 0.399 & $0.632^{\star \star \star *}$ & 0.004 & 674 & 0.472 & $0.641^{* * *}$ & $-0.130^{* \star *}$ \\
\hline \multirow{2}{*}{$\mathrm{SGA} / \mathrm{S}$} & 1 & 674 & 0.019 & $0.138^{\star \star \star}$ & & 674 & 0.122 & $0.349^{* \star *}$ & \\
\hline & 2 & 674 & 0.026 & $0.127^{* \star *}$ & $-0.082^{* *}$ & 674 & 0.127 & $0.381^{* * *}$ & $0.077^{\star}$ \\
\hline \multirow{2}{*}{$\begin{array}{c}\text { OPEXP } \\
\text { /S }\end{array}$} & 1 & 674 & 0.183 & $0.428^{* * *}$ & & 674 & 0.061 & $0.248^{\star \star *}$ & \\
\hline & 2 & 674 & 0.186 & $0.415^{\star \star *}$ & -0.054 & 674 & 0.061 & $0.248^{* * *}$ & 0.001 \\
\hline
\end{tabular}

This study also examined the halo effect on the sustained financial performance of leading IT groups and their control counterparts. Table 6 displays the summarized results. Similar to the data from 2001-2004, the financial performance of firms was substantially associated with prior financial performance. The regression results also partially supported Hypotheses 7 and 8 because nearly two-thirds of the coefficients of IT capability in Table 6 were significant.

\begin{tabular}{|c|c|c|c|c|c|c|c|c|c|}
\hline \multirow[b]{3}{*}{ Ratios } & \multirow[b]{3}{*}{ Model } & \multicolumn{8}{|c|}{2005} \\
\hline & & \multicolumn{4}{|c|}{ Four-digit control group } & \multicolumn{4}{|c|}{ Two-digit control group } \\
\hline & & $\mathbf{N}$ & $R^{2}$ & $\begin{array}{c}\text { Prior year } \\
\text { financial } \\
\text { performance }\end{array}$ & $\begin{array}{c}\text { IT capability } \\
\text { dummy }\end{array}$ & $\mathbf{N}$ & $R^{2}$ & $\begin{array}{c}\text { Prior year } \\
\text { financial } \\
\text { performance }\end{array}$ & $\begin{array}{l}\text { IT capability } \\
\text { dummy }\end{array}$ \\
\hline \multirow{2}{*}{ ROA } & 1 & 648 & 0.155 & $0.393^{\star \star *}$ & & 648 & 0.099 & $0.315^{\star \star \star}$ & \\
\hline & 2 & 648 & 0.171 & $0.365^{\star \star \star}$ & $0.129^{* * *}$ & 648 & 0.123 & $0.276^{\star \star \star}$ & $0.158^{\star \star \star}$ \\
\hline \multirow{2}{*}{ ROS } & 1 & 648 & 0.205 & $0.452^{\star * *}$ & & 648 & 0.453 & $0.673^{* * *}$ & \\
\hline & 2 & 648 & 0.213 & $0.439^{* * *}$ & $0.092^{* * *}$ & 648 & 0.459 & $0.660^{* * *}$ & $0.130^{* * *}$ \\
\hline \multirow{2}{*}{$\mathrm{OI} / \mathrm{A}$} & 1 & 648 & 0.154 & $0.393^{* * *}$ & & 648 & 0.055 & $0.233^{\star * *}$ & \\
\hline & 2 & 648 & 0.175 & $0.360^{* * *}$ & $0.148^{* * *}$ & 648 & 0.091 & $0.185^{\star * *}$ & $0.198^{* * *}$ \\
\hline \multirow{2}{*}{$\mathrm{OI} / \mathrm{S}$} & 1 & 648 & 0.191 & $0.437^{* * *}$ & & 648 & 0.521 & $0.722^{* * *}$ & \\
\hline & 2 & 648 & 0.200 & $0.426^{* * *}$ & $0.091^{* * *}$ & 648 & 0.538 & $0.710^{* * *}$ & $0.128^{\star \star \star *}$ \\
\hline \multirow{2}{*}{$\mathrm{Ol} / \mathrm{E}$} & 1 & 646 & 0.066 & $0.257^{\star \star \star}$ & & 645 & 0.832 & $0.912^{* \star \star}$ & \\
\hline & 2 & 646 & 0.067 & $0.257^{\star * *}$ & -0.033 & 645 & 0.832 & $0.912^{* * *}$ & -0.013 \\
\hline \multirow{2}{*}{$\begin{array}{c}\text { COGS/ } \\
\mathrm{S}\end{array}$} & 1 & 648 & 0.314 & $0.560^{* * *}$ & & 648 & 0.250 & $0.500^{* * *}$ & \\
\hline & 2 & 648 & 0.316 & $0.555^{\star \star \star}$ & -0.048 & 648 & 0.251 & $0.493^{\star * \star}$ & -0.026 \\
\hline $\mathrm{SGA} / \mathrm{S}$ & 1 & 648 & 0.540 & $0.735^{\star * *}$ & & 648 & 0.888 & $0.942^{* * *}$ & \\
\hline
\end{tabular}




\begin{tabular}{|c|c|c|c|c|c|c|c|c|c|}
\hline \multicolumn{10}{|c|}{ Table 6. Results of Tests for Hypotheses 7 and 8} \\
\hline & 2 & 648 & 0.554 & $0.723^{\star \star \star}$ & $-0.118^{\star * *}$ & 648 & 0.890 & $0.939^{* * *}$ & $-0.042^{* \star *}$ \\
\hline \multirow{4}{*}{$\begin{array}{c}\text { OPEXP } \\
\text { /S }\end{array}$} & 1 & 648 & 0.228 & $0.477^{\star \star \star}$ & & 648 & 0.528 & $0.726^{\star \star \star}$ & \\
\hline & 2 & 648 & 0.233 & $0.466^{\star \star \star}$ & $-0.073^{\star \star}$ & 648 & 0.544 & $0.715^{\star \star \star}$ & $-0.129^{* \star *}$ \\
\hline & & \multicolumn{8}{|c|}{2006} \\
\hline & & \multicolumn{4}{|c|}{ Four-digit control group } & \multicolumn{4}{|c|}{ Two-digit control group } \\
\hline Ratios & Model & $\mathbf{N}$ & $R^{2}$ & $\begin{array}{c}\text { Prior year } \\
\text { financial } \\
\text { performance }\end{array}$ & $\begin{array}{c}\text { IT capability } \\
\text { dummy }\end{array}$ & $\mathbf{N}$ & $R^{2}$ & $\begin{array}{c}\text { Prior year } \\
\text { financial } \\
\text { performance }\end{array}$ & $\begin{array}{l}\text { IT capability } \\
\text { dummy }\end{array}$ \\
\hline \multirow{2}{*}{ ROA } & 1 & 626 & 0.001 & 0.038 & & 626 & 0.267 & $0.517^{\star \star \star}$ & \\
\hline & 2 & 626 & 0.009 & 0.019 & $0.091^{* *}$ & 626 & 0.281 & $0.544^{* * *}$ & $-0.121^{* * *}$ \\
\hline \multirow{2}{*}{ ROS } & 1 & 626 & 0.284 & $0.533^{* * *}$ & & 626 & 0.012 & $0.111^{* * *}$ & \\
\hline & 2 & 626 & 0.285 & $0.527^{\star * \star}$ & 0.035 & 626 & 0.013 & $0.115^{\star \star *}$ & -0.022 \\
\hline \multirow{2}{*}{$\mathrm{OI} / \mathrm{A}$} & 1 & 626 & 0.004 & 0.066 & & 626 & 0.204 & $0.451^{* \star *}$ & \\
\hline & 2 & 626 & 0.017 & 0.040 & $0.115^{\star * *}$ & 626 & 0.216 & $0.479^{* * *}$ & $-0.115^{\star * *}$ \\
\hline \multirow{2}{*}{$\mathrm{OI} / \mathrm{S}$} & 1 & 626 & 0.345 & $0.587^{* * *}$ & & 626 & 0.119 & $0.345^{* * *}$ & \\
\hline & 2 & 626 & 0.346 & $0.583^{* * *}$ & 0.029 & 626 & 0.124 & $0.358^{* * *}$ & $-0.069^{*}$ \\
\hline \multirow{2}{*}{$\mathrm{Ol} / \mathrm{E}$} & 1 & 626 & 0.697 & $0.835^{\star \star \star}$ & & 626 & 0.316 & $0.562^{\star * \star}$ & \\
\hline & 2 & 626 & 0.697 & $0.835^{\star \star \star *}$ & -0.009 & 626 & 0.316 & $0.562^{\star * *}$ & 0.010 \\
\hline \multirow{2}{*}{$\begin{array}{c}\mathrm{COGS} / \\
\mathrm{S}\end{array}$} & 1 & 626 & 0.357 & $0.598^{* * *}$ & & 626 & 0.015 & $0.123^{\text {*** }}$ & \\
\hline & 2 & 626 & 0.358 & $0.595^{\star \star \star}$ & -0.029 & 626 & 0.016 & $0.127^{\star \star \star *}$ & 0.022 \\
\hline \multirow{2}{*}{$\mathrm{SGA} / \mathrm{S}$} & 1 & 626 & 0.043 & $0.207^{\star \star \star}$ & & 626 & 0.359 & $0.599^{* \star \star}$ & \\
\hline & 2 & 626 & 0.055 & $0.185^{\star \star \star}$ & $-0.111^{* \star *}$ & 626 & 0.364 & $0.607^{\star \star \star *}$ & $0.070^{\star *}$ \\
\hline \multirow{3}{*}{$\begin{array}{c}\text { OPEXP } \\
\text { IS }\end{array}$} & 1 & 626 & 0.384 & $0.620^{* \star *}$ & & 626 & 0.138 & $0.371^{* \star \star}$ & \\
\hline & 2 & 626 & 0.385 & $0.616^{\star \star \star *}$ & -0.031 & 626 & 0.143 & $0.385^{\star \star \star}$ & $0.074^{*}$ \\
\hline & & \multicolumn{8}{|c|}{2007} \\
\hline & & \multicolumn{4}{|c|}{ Four-digit control group } & \multicolumn{4}{|c|}{ Two-digit control group } \\
\hline Ratios & Model & $\mathbf{N}$ & $R^{2}$ & $\begin{array}{c}\text { Prior year } \\
\text { financial } \\
\text { performance }\end{array}$ & $\begin{array}{c}\text { IT capability } \\
\text { dummy }\end{array}$ & $\mathbf{N}$ & $R^{2}$ & $\begin{array}{c}\text { Prior year } \\
\text { financial } \\
\text { performance }\end{array}$ & $\begin{array}{l}\text { IT capability } \\
\text { dummy }\end{array}$ \\
\hline \multirow{2}{*}{ ROA } & 1 & 592 & 0.010 & $0.102^{\star \star}$ & & 592 & 0.005 & 0.067 & \\
\hline & 2 & 592 & 0.052 & $0.083^{* *}$ & $0.204^{* \star *}$ & 592 & 0.111 & 0.000 & $0.333^{\star * \star}$ \\
\hline \multirow{2}{*}{ ROS } & 1 & 592 & 0.039 & $0.197^{\star \star *}$ & & 592 & 0.267 & $0.517^{\star \star \star}$ & \\
\hline & 2 & 592 & 0.064 & $0.178^{* \star \star}$ & $0.161^{* * *}$ & 592 & 0.303 & $0.437^{* * *}$ & $0.204^{* * *}$ \\
\hline \multirow{2}{*}{$\mathrm{Ol} / \mathrm{A}$} & 1 & 592 & 0.027 & $0.165^{\star \star *}$ & & 592 & 0.010 & $0.100^{* *}$ & \\
\hline & 2 & 592 & 0.064 & $0.140^{\star \star \star}$ & $0.195^{\star \star *}$ & 592 & 0.154 & 0.022 & $0.388^{* * *}$ \\
\hline \multirow{2}{*}{$\mathrm{OI} / \mathrm{S}$} & 1 & 592 & 0.036 & $0.189^{* * *}$ & & 592 & 0.140 & $0.375^{\star \star *}$ & \\
\hline & 2 & 592 & 0.059 & $0.172^{* * *}$ & $0.151^{\text {*** }}$ & 592 & 0.204 & $0.276^{* * *}$ & $0.270^{* * *}$ \\
\hline \multirow{2}{*}{$\mathrm{Ol} / \mathrm{E}$} & 1 & 592 & 0.670 & $0.818^{* * *}$ & & 589 & 0.799 & $0.894^{* * *}$ & \\
\hline & 2 & 592 & 0.671 & $0.817^{* * *}$ & -0.032 & 589 & 0.801 & $0.892^{* * *}$ & $-0.046^{\star *}$ \\
\hline \multirow{2}{*}{$\begin{array}{c}\text { COGS/ } \\
\mathrm{S}\end{array}$} & 1 & 592 & 0.083 & $0.289^{* * \star}$ & & 592 & 0.161 & $0.401^{* * *}$ & \\
\hline & 2 & 592 & 0.095 & $0.278^{* * *}$ & $-0.109^{* * *}$ & 592 & 0.196 & $0.348^{\star * *}$ & $-0.194^{\star * *}$ \\
\hline \multirow{2}{*}{$\mathrm{SGA} / \mathrm{S}$} & 1 & 592 & 0.216 & $0.465^{\star * *}$ & & 592 & 0.204 & $0.451^{* * *}$ & \\
\hline & 2 & 592 & 0.229 & $0.448^{* * *}$ & $-0.114^{* * *}$ & 592 & 0.242 & $0.394^{* * *}$ & $-0.203^{\star * *}$ \\
\hline OPEXP & 1 & 592 & 0.093 & $0.306^{* * *}$ & & 592 & 0.162 & $0.402^{\star \star \star}$ & \\
\hline
\end{tabular}


Table 6. Results of Tests for Hypotheses 7 and 8

\begin{tabular}{|c|c|c|c|c|c|c|c|c|c|}
\hline IS & 2 & 592 & 0.112 & $0.289^{* * *}$ & $-0.138^{* * *}$ & 592 & 0.208 & $0.320^{* * *}$ & $-0.231^{* * *}$ \\
\hline
\end{tabular}

Coefficients of prior ratio and IT capability are standardized.

*** significant at the $1 \%$ level, ${ }^{* *}$ significant at the $5 \%$ level, * significant at the $10 \%$ level

\section{Conclusion and Discussion}

\subsection{Discussion}

We provided evidence that IT capability had a positive association with firm performance using IW 500 data from 2001-2004 contrary to Chae et al. (2014). We considered all firms in the same industry as control groups; whereas Chae et al. (2014) identified a single matched firm as control groups. We believe that it is more appropriate to show that IT leading firms outperform average firms in the same industry, as tested in our study, than to suggest whether an IT leader maintains a superior position over a single rival firm, as examined in Chae et al. (2014). A firm's strategic actions are highly influenced by other companies in the same industry (DiMaggio and Powell, 1983), and industry membership has a critical influence on a firm's profitability (McGahan and Porter, 1997). Especially, when a firm is exposed to uncertainty, the firm is likely to decide its strategic posture, which means the intent of a strategy relative to the current and future state of an industry, by following other firms' behaviors in the same industry instead of observing a rival firm's intention (Courtney et al., 1997). Similarly, when a firm invests in IT, the firm considers industry average rather than a firm's peers (Mithas et al., 2013). In this sense, it is desirable to compare a firm's performance with industry average rather than a specific peer.

We need to apprehend what factors caused the disparate result between Chae et al. (2014) and this research. The mode of selecting the control group (i.e., a matched firm or all firms in the same industry) seems a noticeable factor, which brings about the inconsistent results. However, the possible selection bias from the choice of a single benchmark group (Santhanam and Hartono, 2003) cannot be a serious concern because Chae et al. (2014) considered 296 leader-control pairs in four years, and the sample size was large enough.

Instead, there must be moderating or mediating factors that we did not consider. For instance, the firm size of control groups might have influenced firm performance. The firms in control groups investigated in Chae et al. (2014) were generally bigger than the firms in the control groups in this study. A large body of prior research has shown that firm size has an effect on firm performance such as ROA or Tobin's $q$ (Wiggins and Ruefli, 2002). Also, the utilization of IT is closely related to firm size (Atasoy et al., 2016). Thus, the size of control groups may have had an effect on the relationship between IT capability and firm performance.

Another possible cause for the discrepancy could be connected to the industry factors. Kohli and Devaraj (2003) proved that the type of industry had a significant moderating impact on the payoff metric. Mithas et al. (2012) showed that IT has a greater effect on firm profitability in service industries than in manufacturing industries. We may need to investigate the impact of IT capability on firm performance according to the industry sectors rather than aggregate the impact to understand the mixed results.

Bhadadwaj (2000) has been cited more than 3,500 times according to Google Scholar. The reason that the paper has such an influential position is that it provided a theoretical background to show how and why IT contributes to firm performance. Without using the concept of IT capability, it is still elusive to link IT to the economic value of a firm (e.g., profitability). Bharadwaj (2000) suggested that IT capabilities, IT resource with the ability to use them, are idiosyncratic and firm-specific resources that are not transferable to other firms and contribute to the competitiveness of firms. During the last 16 years, there were substantial changes and improvements in IT. Enterprise resource planning, e-commerce, customer relationship management, and knowledge management were introduced after the mid-1990s (Wang, 2010). It can be argued that the concept of IT capability is outdated in the sense that the construct does not reflect the newly developed IT systems. A specific method for measuring IT capability can be evolved over time and has changed to include the changing nature of IT (Lee et al., 2015; Lu and Ramamurthy, 2011). However, this study suggests that the core tenet that IT capability is the source of competitive does not change. This is the implication for the practitioners. A firm's IT applications and its ability to use them are still critical to outperform its rivals in the same industry. 
We would argue the necessity of replicating studies in the IS research area. As Dennis and Valacich (2015) stated, "replication is one of the main principles of the scientific method. [...] Replication will either improve confidence in our research findings or identify important boundary conditions." (p. 1) To expand our understanding of a certain topic in the IS area, we need to retest and affirm the research results of prior studies. Particularly, when mixed findings are uncovered, it becomes difficult to overemphasize the need for replications. Replication studies do not have limited theoretical contributions in the sense that a theory is iteratively improved and elaborated by developing conceptual models and supporting the models with empirical results. Also, replications are necessary to increase the generalizability of research models to other research settings (Compeau et al., 2012).

\subsection{Conclusion}

This study replicated and extended three prior MIS Quarterly studies on the contribution of IT capability on firms' financial performance. Bharadwaj (2000) and Santhanam and Hartono (2003) evidenced the positive association between IT capability and firm performance based on IW 500 data from 1991-1994. In contrast, Chae et al. (2014) identified no relationship between IT capability and firm performance using IW 500 data from 2001-2004. The present study similarly analyzed IW 500 list from 2001-2004 but compared the financial performance of leading IT groups with that of all firms in the same industry, as accomplished by Santhanam and Hartono (2003). Interestingly, contrary to the results reported by Chae et al. (2014), we observed a positive impact of IT capability on firms' financial performance. We do not argue that the procedure of Chae et al. (2014) or their research results were inappropriate; instead, we contend that the research findings can be differentiated depending on the selected control group. According to the results of this study, IT capability played a critical role in developing the competitive advantage of firms and was an advantage that could be sustained over subsequent years. 


\section{References}

Atasoy, H., Banker, R. D., \& Pavlou, P. A. (2016). On the long term effects of IT use on firm-level employment. Information Systems Research, 27(1), 6-26.

Bharadwaj, A. S. (2000). A resource-based perspective on information technology capability and firm performance: An empirical investigation. MIS Quarterly, 24(1), 169-196.

Bhatt, G. D., \& Grover, V. (2005). Types of information technology capabilities and their role in competitive advantage: An empirical study. Journal of Management Information Systems, 22(2), 253-277.

Chae, H.-C., Koh, C. E., \& Prybutok, V. R. (2014). Information technology capability and firm performance: Contradictory findings and their possible causes. MIS Quarterly, 38(1), 305-326.

Compeau, D., Marcolin, B., Kelley, H., \& Higgins, C. (2012). Research commentary-generalizability of information systems research using student subjects-A reflection on our practices and recommendations for future research. Information Systems Research, 23(4), 1093-1109.

Courtney, H., Kirkland, J., \& Viguerie, P. (1997). Strategy under uncertainty. Harvard Business Review, 75(6), 67-79.

Dennis, A. R., \& Valacich, J. S. (2015). A replication manifesto. AIS Transactions on Replication Research, 1, 1-5.

DiMaggio, P., \& Powell, W. W. (1983). The iron cage revisited: Collective rationality and institutional isomorphism in organizational fields. American Sociological Review, 48(2), 147-160.

Fama, E. F., \& French, K. R. (2000). Forecasting profitability and earnings. Journal of Business, 73(2), 161-175.

Kohli, R., \& Devaraj, S. (2003). Measuring information technology payoff: A meta-analysis of structural variables in firm-level empirical research. Information Systems Research, 14(2), 127-145.

Lee, O. K., Sambamurthy, V., Lim, K. H., \& Wei, K. K. (2015). How does IT ambidexterity impact organizational agility? Information Systems Research, 26(2), 398-417.

Lu, Y., \& Ramamurthy, K. (2011). Understanding the link between information technology capability and organizational agility: An empirical examination. MIS Quarterly, 35(4), 931-954.

McGahan, A. M., \& Porter, M. E. (1997). How much does industry matter, really? Strategic Management Journal, 18(S), 15-30.

Mithas, S., Tafti, A. R., Bardhan, I., \& Goh, J. M. (2012). Information technology and firm profitability: Mechanisms and empirical evidence. MIS Quarterly, 36(1), 205-224.

Mithas, S., Tafti, A. R., \& Mitchell, W. (2013). How a firm's competitive environment and digital strategic posture influence digital business strategy. MIS Quarterly, 37(2), 511-536.

Santhanam, R., \& Hartono, E. (2003). Issues in linking information technology capability to firm performance. MIS Quarterly, 27(1), 125-153.

Stiglitz, J. E. (1989). Incentives, information, and organizational design. Cambridge, MA: National Bureau of Economic Research.

Stoel, M. D., \& Muhanna, W. A. (2009). IT capabilities and firm performance: A contingency analysis of the role of industry and IT capability type. Information \& Management, 46(3), 181-189.

Tanriverdi, H., \& Uysal, V. B. (2015). When IT capabilities are not scale-free in merger and acquisition integrations: How do capital markets react to IT capability asymmetries between acquirer and target? European Journal of Information Systems, 24(2), 145-158.

Wang, N., Liang, H., Zhong, W., Xue, Y., \& Xiao, J. (2012). Resource structuring or capability building? An empirical study of the business value of information technology. Journal of Management Information Systems, 29(2), 325-367.

Wang, P. (2010). Chasing the hottest IT: Effects of information technology fashion on organizations. MIS Quarterly, 34(1), 63-85. 
Wiggins, R. R., \& Ruefli, T. W. (2002). Sustained competitive advantage: Temporal dynamics and the incidence and persistence of superior economic performance. Organization Science, 13(1), 81-105. 


\section{Appendix A: IT Capability and InformationWeek 500 list}

This research and three prior MIS Quarterly studies measured IT capability by utilizing InformationWeek (IW) list. Prior studies mentioned that the criteria for identifying IT leading firms have changed, leading to inconsistent research results about the relationship between IT capability and firm performance (Chae et al., 2014, Santhanam and Hartono, 2003). We have reviewed the criteria used by InformationWeek in the early 1990s and 2000s. We have confirmed that there was a change in the criteria as summarized in Table A-1. In 1991-1994, IT leaders were identified based on the value of installed IT applications within firms or the revenue of the firms. On the other hand, the focus was given to the effective and efficient use of IT as well as innovative applications when IW editors selected IT leaders in 2001-2004. Therefore, it is not appropriate to compare directly the results of Bharadwaj (2000) and Santhanam and Hartono (2003) with the conclusion of Chae et al. (2014). However, our study and Chae et al. (2014) are using the same criteria, which were consistent in 2001-2004.

\begin{tabular}{|c|l|c|}
\hline \multicolumn{3}{|c|}{ Table A-2. The Criteria for Identifying IT Leading Firms } \\
\hline Years & \multicolumn{1}{|c|}{ Criteria } & \multicolumn{1}{|c|}{ Source } \\
\hline $\begin{array}{c}1991- \\
1992\end{array}$ & IT leaders are ranked based on the value of their installed base of technology. & $\begin{array}{c}\text { Chae et al. (2014) and } \\
\text { InformationWeek } \\
(1991 ; 1992) 1^{1}\end{array}$ \\
\hline $\begin{array}{c}1993- \\
1994\end{array}$ & $\begin{array}{l}\text { Revenue and the use of technology were the key criteria for identifying IT } \\
\text { leaders. }\end{array}$ & $\begin{array}{c}\text { Chae et al. (2014) and } \\
\text { InformationWeek } \\
(1993 ; 1994)^{2}\end{array}$ \\
\hline $\begin{array}{c}2001- \\
2004\end{array}$ & $\begin{array}{l}\text { InformationWeek editors identified innovative IT organizations based in the } \\
\text { U.S. that demonstrated a pattern of technological, procedural, and } \\
\text { organizational innovation. Firms are known to have successfully launched } \\
\text { innovative or strategic applications tend to be ranked as the leaders. }\end{array}$ & $\begin{array}{c}\text { Chae et al. (2014) and } \\
\text { InformationWeek } \\
(2001 ; 2002)^{3}\end{array}$ \\
\hline
\end{tabular}

Another concern of using IW 500 is whether the criteria reflect the conceptual definition of IT capability. IT capability encompasses three concepts: IT infrastructure, the managerial ability of IT, and intangible assets in IT applications (Bharadwaj, 2000). It seems that the criteria used in 1991-1992 did not fully represent the definition of IT capability since they were more concentrated on the IT infrastructure; the criteria in 1993-1994 did not measure IT but assessed a firm's financial performance. However, the criteria used for IW in 2001-2004 have a good fit for the definition of IT capability because the technological innovation assesses IT infrastructure, procedural innovation describes intangible aspect such as business processes, and organizational innovation evaluates the managerial aspect of IT. IW 500 list can be used as a proxy for IT capability although survey approach (Bhatt and Grover, 2005; Lu and Ramamurthy, 2011) may complement the limit of secondary data. Other secondary variables for IT such as IT expenditure, IT assets, and IT capital may not be proper indices to measure IT capability since they only considered only one aspect of IT capability.

\footnotetext{
${ }_{1}^{1}$ InformationWeek, Sep. 16, 1991; Sep. 21, 1992

2 InformationWeek, Sep. 27, 1993; Oct. 10, 1994

${ }^{3}$ InformationWeek, Sep. 7, 2001; Sep. 23, 2002
} 


\section{About the Authors}

Inmyung Choi is a Ph.D. student in the Department of Supply Chain and Information Systems, the College of Business at lowa State University. Prior to his doctoral studies, he received his M.S. degree in Management Engineering from Korea Advanced Institute of Science and Technology (KAIST) Business School. His research focuses on examining the role of information technology in improving the competitiveness of firms based on econometrics, text analysis, and survey. He has presented his research at several conferences such as the Americas Conference on Information Systems and the Academy of Management Annual Meeting.

Joey F. George is a professor of Information Systems and the John D. Devries Endowed Chair in Business in the College of Business at lowa State University. He received his doctorate in management from the University of California at Irvine in 1986 and his bachelor's degree in English from Stanford University in 1979. His research interests focus on the use of information systems in the workplace, including deceptive computer-mediated communication, computer-based monitoring, and group support systems. He is a former president of AIS, and AIS fellow, and recipient of the LEO Lifetime Achievement Award.

Copyright (C) 2016 by the Association for Information Systems. Permission to make digital or hard copies of all or part of this work for personal or classroom use is granted without fee provided that copies are not made or distributed for profit or commercial advantage and that copies bear this notice and full citation on the first page. Copyright for components of this work owned by others than the Association for Information Systems must be honored. Abstracting with credit is permitted. To copy otherwise, to republish, to post on servers, or to redistribute to lists requires prior specific permission and/or fee. Request permission to 
publish from: AIS Administrative Office, P.O. Box 2712 Atlanta, GA, 30301-2712 Attn: Reprints or via email from ais@aisnet.org. 